\title{
Survey towards Masked Face Detection for Pandemic
}

\author{
Yash Agrawal \\ Dept. of Computer Engineering \\ JSPM's, BSIOTR, Wagholi, Pune, \\ India
}

\author{
Diksha Nitnaware \\ Dept. of Computer Engineering \\ JSPM's, BSIOTR, Wagholi, Pune, \\ India
}

\author{
Gauri Kulkarni \\ Dept. of Computer Engineering \\ JSPM's, BSIOTR, Wagholi, Pune, \\ India
}

\author{
Saurabh Patil \\ Dept. of Computer Engineering \\ JSPM's, BSIOTR, Wagholi, \\ Pune, India
}

\author{
Archana Lomte, PhD \\ Dept. of Computer Engineering \\ JSPM's, BSIOTR, Wagholi, \\ Pune, India
}

\begin{abstract}
Covid19 has given another personality for wearing a veil. It is significant when these covered countenances are recognized precisely and proficiently. As an exceptional face recognition task, face veil identification is substantially more troublesome on account of outrageous impediments which prompt the deficiency of face subtleties. Furthermore, there is basically no current enormous scope precisely marked concealed face dataset, which increments the trouble of face veil discovery. The framework urges to utilize $\mathrm{CNN}$-based profound learning calculations which have done tremendous advancement towards explores in face identification. In this paper, propose a novel CNN-based technique that is shaped by three convolutional neural organizations to recognize the face veil. Plus, in view of the deficiency of face veiled preparing tests, propose another dataset called" face cover dataset" to tweak CNN models. Assess proposed face veil recognition calculation on the face cover testing set, and it accomplishes agreeable execution.
\end{abstract}

\section{Keywords}

Face Mask, CNN, Face Detection, Deep Learning.

\section{INTRODUCTION}

Towards the finish of this decade, the face has another distinguishing proof because of ascend in COVID-19 cases. Coronavirus is an infection that gets exacted when a tainted individual interacts with some other individual. A contaminated individual can leave hints of infection on things around them. His spit or contact is seen to be the most irresistible medium to convey this infection. Subsequently, a method of insurance individuals all around need to wear face cover to forestall themselves and everybody around them to get tainted by the VIRUS. This control has been made necessary which is helping in checking the COVID-19 cases. The government has used severe laws for wearing veils when around there. Individuals are fined for not wearing cover or not wearing it appropriately. Remembering the need for the time have proposed a technique for distinguishing the cover on the face.

The paper proposes a stage savvy strategy to identify the square of the face in pictures and observation recordings. The following stage includes the identification of the veil on this square. Further advances include if the cover is worn appropriately. The initial step of face identification is one of the longest-explored PC vision issues, which can be followed back about 50 years prior. In any case, the majority of the early face recognition calculations can't meet the functional need. The Viola-Jones as a first specialist for face discovery has a face identifier that comprises a progression of classifiers going from easy to complex ones. Later scientists kept on considering it dependent on it, and large numbers of them apply more perplexing and spellbinding highlights to make the identifier all the more impressive.

As of late, profound learning has made extraordinary forward leaps in numerous PC vision zones, for example, general item discovery, object order, object division, and obviously, face identification. Profound learning doesn't have to physically configuration highlights, as the CNN (Convolutional Neural Networks) can consequently take taking invaluable highlights from the preparation pictures. The proposed framework includes utilization of $\mathrm{CNN}$ for face location and discovery of veil at a later stage with K-implies. The framework will help distinguish individuals wearing veil also individuals not wearing cover or individuals who have not worn veil as expected. This is course will assist with carrying control to the public also insurance through which they can without much of a stretch wander around with cover out in the open spot and help check the COVID-19 contamination.

\section{LITERATURE SURVEY \\ 2.1 Multi-scale feature extraction for single face recognition}

The Single example face acknowledgment has consistently been a hot yet troublesome issue in face acknowledgment. By considering choosing hearty highlights and creating virtual examples all the while, the paper proposes a multi-scale support vector change (MSSVT) based technique to produce multi-scale virtual examples for single picture acknowledgment. The strategies to take care of issue are separated into two classifications. One is to search for and select highlights that are powerful to the quantity of tests, according to the perspective of highlight choice, like PCA and 2DPCA. However, when every individual has just one face to be prepared, the component data separated from the element extraction calculation will likewise be exceptionally restricted, bringing about an awful acknowledgment execution. The other is to create numerous virtual examples according to the perspective of the all-encompassing example, along these lines lessening the effect of the example size. [3] 


\subsection{Face recognition method based on sparse representation and feature fusion}

The creators propose a multi-highlight combination face acknowledgment strategy dependent on meagre portrayal. The centre's thought is to discover the inadequacy through preparing, and afterward utilize the inadequate coefficient and preparing tests to address the test tests, and afterward, the ideal scanty arrangement is acquired by tackling the 11standard issue. The acknowledgment consequences of the highlight combination technique are superior to any single component calculation under the state of non-impediment or impediment. When there are under 10 photos of every class of individuals in the preparation test and the impediment type isn't controllable, calculation can in any case acquire a high acknowledgment rate. [5]

\subsection{Spatial pyramid pooling in deep convolutional networks for visual recognition}

For Visual Recognition, Scales, Sizes, and Aspect Ratios are considered significant factors. SPP (Spatial Pyramid Pooling) is an adaptable answer for dealing with these components. In the setting of profound organizations, these elements have gotten less thought, accordingly, the framework is prepared with profound layer networks considering the SPP layer. SPPnet shows extraordinary exactness in arrangement/recognition assignments and enormously speeds up DNN-based identification. Their investigations additionally show that many time-demonstrated procedures/experiences in PC vision can in any case assume significant parts in profound organizations-based acknowledgment. [1]

\subsection{Face and Gender Recognition System Based on Convolutional Neural networks}

Author name: Y. Zhou, H. Ni, F. Ren and X. Kang

The proposed Face and Gender Recognition System understands the mix of picture face acknowledgment and sexual orientation acknowledgment module, which empowers face acknowledgment as well as gender acknowledgment in a complex foundation. In light of the ResNet50 neural organizations, utilize the worldwide normal pool (GAP) rather than the completely associated layer before conclusive yield, trailed by the softmax layer, which diminished the size of the organizations. By developing a particularly basic construction, the precision of the framework acknowledgment has been improved. [6]

\subsection{Dynamic Feature Matching on Partial Face Recognition}

Description: In The halfway face acknowledgment is having application in an expansive range of various fields. The various methodologies utilized for the incomplete face acknowledgment are the central issue-based methodology, district-based methodology, and $\mathrm{CNN}$-based methodology. In key point-based, the well-known strategy was MKD-SRC. In the locale-based halfway face acknowledgment approach, the noticeable model is MR-CNN. Amidst various methodologies in halfway face acknowledgment, it is inferred that the CNNbased methodologies are the nearly best methodology. The current novel methodology proposed for incomplete face acknowledgment. In CNN-based is called Dynamic Feature Matching (DFM). The unique component word reference connecting to the test is accomplished. DFM can yield the upsides of the properties of FCN and produce recognizing highlights all the more exactly. DFM is having a promising application in different video acknowledgment approaches later on. [2]

\subsection{Implementation of Principal Component Analysis on Masked and Non-masked Face Recognition}

The paper examinations non-concealed face acknowledgment and covered face acknowledgment precision utilizing Principal Component Analysis (PCA) to perceive an individual. It demonstrated that a face without cover gives a better acknowledgment rate in PCA based face acknowledgment framework. In any case, when an individual is wearing a cover, facial acknowledgment gives a helpless acknowledgment rate. It is discovered that extricating highlight from a veiled face is not exactly a non-covered face. In view of missing highlights for wearing a veil which declines the acknowledgment rate. At last, it is inferred that customary factual calculation Principal Component Analysis (PCA) is better for typical face acknowledgment however not for covered face acknowledgment. Thus, later on, worry to improve the precision of covered face acknowledgment utilizing other complex AI strategies. [7]

\section{EXISTING SYSTEM}

The previous frameworks planned have taken measurable strategies for facial acknowledgment. Later framework overviewed has utilized component extraction as a strategy for facial acknowledgment. In papers, for example, [3] and [5], different component extraction is utilized with the inadequate portrayal. The papers [1] [6] utilize CNN as the most recent system for facial acknowledgment. Set forth study CNN as an essential structure that will be utilized in the proposed framework.

The customary neural organization isn't fit for managing pictures. Consider in the event of the ordinary organization, envision every pixel is associated with one neuron and there will be a great many neurons which will be computationally costly. Convolutional Neural Network (CNN) handles pictures in an unexpected way, yet, it follows the overall idea of Neural Network. In developing the CNN, it basically comprises of three sections - Convolution, Polling, and Flattering. The basic reason for convolution is to choose qualities from the information picture. It moderates the spatial connection between pixels by learning picture qualities utilizing little squares of info information. The yield got is a lattice known as the element map. A further activity called 


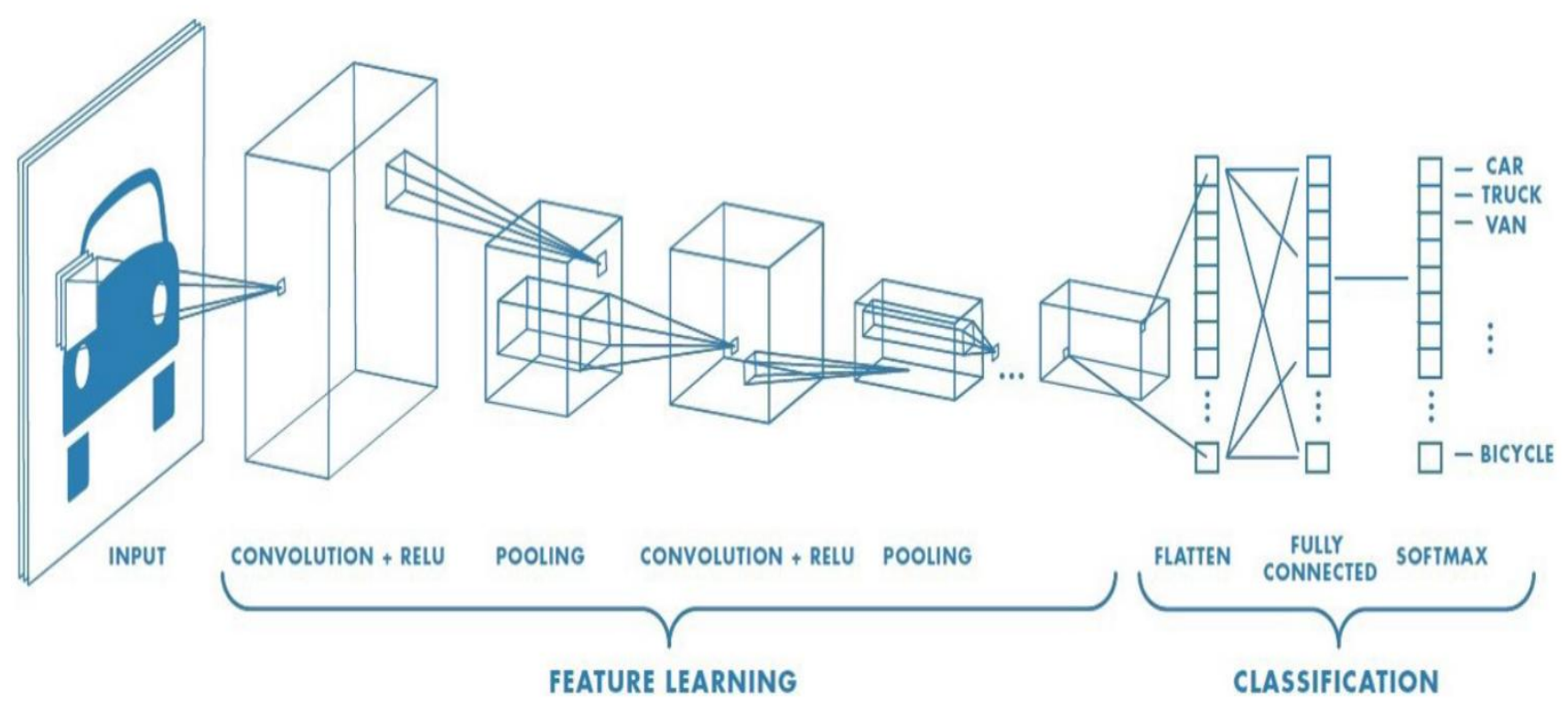

Fig 1: CNN

ReLU is utilized after each convolution activity. The subsequent stage is pooling which is otherwise called subtesting or down-examining. Pooling lessens the length of each element map yet keeps up the main data. In Max Pooling, it characterizes a spatial area and takes the greatest component from the corrected element map inside that window. The other strategy is to take the normal of all components around there. In the wake of pooling, the next stage comes is levelling. In this progression, the framework is changed over into a straight exhibit so that to include it into the hubs of the neural organization. The full association is interfacing a convolutional organization to a neural organization and afterward ordering organization. The utilization of CNN helps in limiting the number of boundaries required for pictures.

Coronavirus circumstance has led to assurance of face wearing a veil. The Papers [2] [7] let us study the fractional or concealed face acknowledgment with the utilization of CNN.

\section{CONCLUSION}

The study shows that these frameworks are the most valuable utility as of late to recognize the cover on the face. The model examinations for face recognition and further to recognize the person who is not wearing can be used to stop the spread of infection. As a social reason, this framework can be carried out at shopping centers also at public spots to control the spread of infection. For future work, a similar framework can be used for the personality of the face with face cover in which more highlights can be considered from the facial district.

\section{REFERENCES}

[1] Kaiming He, Xiangyu Zhang, Shaoqing Ren, and Jian Sun. Spatial pyramid pooling in deep convolutional networks for visual recognition. In European Conference on Computer Vision, pages 346-361. Springer,2014

[2] Wei Bu, Jiangjian Xiao, Chuanhong Zhou, Minmin Yang, Chengbin Peng A Cascade Framework for Masked Face Detection, IEEE 8th International Conference on CIS \& RAM, Ningbo, China, 2017.

[3] X. Xu, L. Zhang and F. Li, "MSSVT: Multi-scale feature extraction for single face recognition," 2018 24th International Conference on Pattern Recognition (ICPR),
Beijing, 2018, pp. 1996-2001, doi: 10.1109/ICPR.2018.8545343.

[4] Lun Zhang, Rufeng Chu, Shiming Xiang, Shengcai Liao, and Stan Z Li. Face detection based on multi-block lbp representation. In International Conference on Biometrics, pages 11-18. Springer, 2007.NaliniPriya G, Priyadarshani P, RajaRajeshwari K, IEEE 6thInternational Conference on smart structures and systems ICSSS 2019.

[5] C. Jiang, M. Wang, X. Tang and R. Mao, "Face recognition method based on sparse representation and feature fusion," 2019 Chinese Automation Congress (CAC), Hangzhou, China, 2019, pp. 396-400, doi: 10.1109/CAC48633.2019.8997456.

[6] Y. Zhou, H. Ni, F. Ren and X. Kang, "Face and Gender Recognition System Based on Convolutional Neural networks," 2019 IEEE International Conference on Mechatronics and Automation (ICMA), Tianjin, China, 2019, pp. 1091-1095, doi: 10.1109/ICMA.2019.8816192.

[7] M. R. Reshma and B. Kannan, "Approaches on Partial Face Recognition: A Literature Review," 2019 3rd International Conference on Trends in Electronics and Informatics (ICOEI), Tirunelveli, India, 2019, pp. 538544, doi: 10.1109/ICOEI.2019.8862783.

[8] M. S. Ejaz, M. R. Islam, M. Sifatullah and A. Sarker, "Implementation of Principal Component Analysis on Masked and Non-masked Face Recognition," 2019 1st International Conference on Advances in Science, Engineering and Robotics Technology (ICASERT), Dhaka, Bangladesh, 2019, pp. 1-5, doi: 10.1109/ICASERT.2019.8934543."

[9] E. Winarno, I. Husni Al Amin, H. Februariyanti, P. W. Adi, W. Hadikurniawati and M. T. Anwar, "Attendance System Based on Face Recognition System Using CNNPCA Method and Real-time Camera," 2019 International Seminar on Research of Information Technology and Intelligent Systems (ISRITI), Yogyakarta, Indonesia, 2019, pp. 301-304, doi: 10.1109/ISRITI48646.2019.9034596. 
[10] S. Sawhney, K. Kacker, S. Jain, S. N. Singh and R. Garg, "Real-Time Smart Attendance System using Face Recognition Techniques," 2019 9th International Conference on Cloud Computing, Data Science \& Engineering (Confluence), Noida, India, 2019, pp. 522525, doi: 10.1109/CONFLUENCE.2019.8776934.
[11] W. Zeng, Q. Meng and R. Li, "Design of Intelligent Classroom Attendance System Based on Face Recognition," 2019 IEEE 3rd Information Technology, Networking, Electronic and Automation Control Conference (ITNEC), Chengdu, China, 2019, pp. 611615, doi: 10.1109/ITNEC.2019.8729496. 